\title{
Hypoxic alligator embryos: Chronic hypoxia, catecholamine levels and autonomic responses of in ovo alligators
}

\author{
John Eme, Jordi Altimiras, James W Hicks and Dane A. Crossley II
}

\section{Linköping University Post Print}

N.B.: When citing this work, cite the original article.

Original Publication:

John Eme, Jordi Altimiras, James W Hicks and Dane A. Crossley II, Hypoxic alligator embryos: Chronic hypoxia, catecholamine levels and autonomic responses of in ovo alligators, 2011, Comparative Biochemistry and Physiology - Part A: Comparative Physiology, (160), 3, 412-420.

http://dx.doi.org/10.1016/j.cbpa.2011.07.010

Copyright: Elsevier 
Hypoxic Alligator Embryos: Chronic hypoxia, catecholamine levels and autonomic responses of in ovo alligators

John Eme ${ }^{1 *}$, Jordi Altimiras ${ }^{2}$, James W. Hicks ${ }^{3}$, Dane A. Crossley II ${ }^{1}$

${ }^{1}$ Department of Biological Sciences, University of North Texas, Denton, TX 76203, USA

${ }^{2}$ Department of Biology, IFM, Linköpings Universitet, SE-58183, Linköping, Sweden

${ }^{3}$ Department of Ecology and Evolutionary Biology, University of California Irvine, Irvine CA 92697, USA

\begin{abstract}
Abbreviations
CAM - Chorioallantoic Membrane

$\mathrm{f}_{\mathrm{H}}-$ heart rate (beats $\mathrm{min}^{-1}$ )

$\mathrm{H} 10$ - alligator embryos artificially incubated in chronic hypoxia $\left(10 \% \mathrm{O}_{2}\right)$, beginning at $20 \%$ of development

MAP - Mean Arterial Pressure $(\mathrm{kPa})$ in a CAM artery

$\mathrm{N} 21$ - alligator embryos artificially incubated in chronic normoxia $\left(21 \% \mathrm{O}_{2}\right)$, beginning at $20 \%$ of development
\end{abstract}

\footnotetext{
*Corresponding author

John Eme, Ph.D.

http://web.mac.com/jeme

University of North Texas

Department of Biological Sciences

1155 Union Circle, \#305220

Denton, TX 76203-5017

USA

E-mail address: johneme34@gmail.com
} 


\begin{abstract}
Hypoxia is a naturally occurring environmental challenge for embryonic reptiles, and this is the first study to investigate the impact of chronic hypoxia on the in ovo development of autonomic cardiovascular regulation and circulating catecholamine levels in a reptile. We measured heart rate $\left(\mathrm{f}_{\mathrm{H}}\right)$ and chorioallantoic arterial blood pressure (MAP) in normoxic ('N21') and hypoxic-incubated ('H10'; 10\% $\mathrm{O}_{2}$ ) American alligator embryos (Alligator mississippiensis) at 70, 80 and 90\% of development. Embryonic alligator responses to adrenergic blockade with propranolol and phentolamine were very similar to previously reported responses of embryonic chicken, and demonstrated that embryonic alligator have $\alpha$ and $\beta$-adrenergic tone over the final third of development. However, adrenergic tone originates entirely from circulating catecholamines and is not altered by chronic hypoxic incubation, as neither cholinergic blockade with atropine nor ganglionic blockade with hexamethonium altered baseline cardiovascular variables in N21 or H10 embryos. In addition, both atropine and hexamethonium injection did not alter the generally depressive effects of acute hypoxia - bradycardia and hypotension. However, H10 embryos showed significantly higher levels of noradrenaline and adrenaline at $70 \%$ of development, as well as higher noradrenaline at $80 \%$ of development, suggesting that circulating catecholamines reach maximal levels earlier in incubation for H10 embryos, compared to N21 embryos. Chronically elevated levels of catecholamines may alter the normal balance between $\alpha$ and $\beta$-adrenoreceptors in $\mathrm{H} 10$ alligator embryos, causing chronic bradycardia and hypotension of $\mathrm{H} 10$ embryos measured in normoxia.
\end{abstract}

Keywords:

Adrenergic tone

Alligator

Cholinergic tone

Development

Embryo

Hypoxia

Reptile 


\section{Introduction}

Interest in embryonic cardiovascular regulation during in ovo development has increased over the past 20 years. These studies have been primarily confined to embryonic chickens, but recent work has expanded to investigate species that may routinely experience more extreme natural environmental variations in developmental conditions, such as reptiles (e.g. Corona and Warburton, 2000; Crossley and Altimiras, 2005; Eme et al., 2011a,b). Hypoxic conditions are known to be present in reptilian nests during development, but data on the cardiovascular implications of this condition for the developing animal are limited (Corona and Warburton, 2000; Crossley and Altimiras, 2005; Eme et al., 2011a,b). Many reptiles, including the American alligator (Alligator mississippiensis), lay eggs underground or in mound nests, and under these conditions the incubating eggs may be exposed to both acute and chronic hypoxia (Lutz and Dunbar-Cooper, 1984; Seymour et al., 1986; Booth, 1998; Booth, 2000). Recorded minimum percent oxygen values within reptile nests have reached 11-15\% (Ackerman, 1980; Lutz and Dunbar-Cooper, 1984; Miller, 2008), with hypoxia likely resulting from several factors, including changes in gas conductance within the nest, rising egg metabolism and/or metabolic activity of nest microorganisms (Kam, 1993). As in birds, gases are exchanged between the atmosphere and the reptilian embryo through the chorioallantoic membrane (CAM) vasculature, a specialized vascular membrane that lies directly beneath the eggshell and outermost shell membrane. Chronic regional hypoxia increases vascularization of the CAM in alligators (Corona and Warburton, 2000), and this accompanied addition of parallel vascular beds could be a mechanism leading to an observed decrease in chorioallantoic resistance and pressure (Crossley and Altimiras, 2005). This decreased pressure could account for increased blood flow to the CAM in chronic hypoxic alligator embryos, compared to normoxic embryos (Eme et al., 2011a). Therefore, the cardiovascular system of the developing reptilian embryo is susceptible and responsive (plastic) to changes in nest atmospheric gas composition.

In chronically hypoxic-incubated alligator embryos, putative mechanisms exist for the observed reduction in arterial pressure - increased CAM vascularization (Corona and Warburton, 2000), as well as the observed increased blood flow to the CAM - ventricular hypertrophy and decreased CAM pressure (Crossley and Altimiras, 2005; Eme et al., 2011a). However, the mechanism(s) that underlie alligator embryo's documented bradycardia in response to acute hypoxia $\left(10 \% \mathrm{O}_{2}\right)$, as well as the bradycardia measured in normoxia $\left(21 \% \mathrm{O}_{2}\right)$ for embryos incubated in chronic hypoxia $\left(10 \% \mathrm{O}_{2}\right)$ remain in question (Crossley and Altimiras, 2005) and could be caused by altered nervous control and/or humoral control. In chicken embryos (Gallus gallus), chronic hypoxic incubation $\left(15 \% \mathrm{O}_{2}\right)$ can cause sympathetic hyperinnervation (Ruijtenbeek et al., 2009), and embryonic chickens respond to hypoxia with an $\alpha$ - 
adrenergic-mediated redistribution of cardiac output, preferentially perfusing the brain, heart and CAM (Mulder et al., 1988; Mulder et al., 2001; Crossley et al., 2003b). Embryonic chickens chronically incubated in hypoxia $\left(14-15 \% \mathrm{O}_{2}\right)$ show a bradycardia and hypotension, similar to alligators (Crossley and Altimiras, 2005). However, the hypotension is unrelated to the bradycardia and is likely caused by elevated levels of circulating catecholamines acting on vascular beds with exclusively $\beta$-adrenoreceptors or acting on $\beta$-adrenoreceptors with altered sensitivities (Lindgren et al., 2011). In addition, circulating catecholamines cause potentially competing responses within the chicken egg, with vasodilation in the extra-embryonic CAM vasculature (Lindgren et al., 2010; Lindgren et al., 2011) and vasoconstriction in the intra-embryonic vasculature (Lindgren et al., 2011).

It is possible that chronic hypoxia accelerates the onset or development of alligator embryos' autonomic nervous system (ANS) or catecholamine-based control over heart rate and/or blood pressure, possibly through competing responses caused by increased circulating catecholamines. For example, higher chronic levels of circulating catecholamines could be caused by increased secretion from neuronal vesicles whose development has been accelerated. In addition, the bradycardia observed during acute hypoxia or for chronically hypoxic alligator embryos could be caused by transitory or earlier development of vagal tone, respectively. The purpose of this study was to investigate the mechanisms that contribute to changes in heart rate and blood pressure in embryonic alligators exposed to chronic hypoxia. Our particular focus was on the maturation of the tonic adrenergic and cholinergic control of the cardiovascular system as well as changes in circulating catecholamine levels. We expected that chronic hypoxic incubation would accelerate the onset of cholinergic and/or adrenergic tone and increase circulating catecholamines in embryonic alligators.

\section{Materials and methods}

\subsection{Alligator embryo acquisition and incubation}

American alligator eggs (Alligator mississippiensis) were obtained from the Rockefeller Wildlife Refuge in Grand Chenier, LA, USA and transported by air or automobile to a lab. Eggs from each clutch were staged to determine each clutches' post-laying time, as previously described (Ferguson 1985; Crossley et al. 2003c; $72 \mathrm{~d}$ total incubation period at $30^{\circ} \mathrm{C}$ ). Embryos were incubated at $30{ }^{\circ} \mathrm{C}$ in either a walk-in, constant temperature room or a reptile egg incubator (Percival Scientific Inc. Perry, IA, USA), ensuring that all embryos were developing as females. All embryos were incubated in plastic containers 
containing a 2:1, vermiculite:water mixture. Water content of the vermiculite, determined by mass at the beginning of incubation, was maintained by weighing the box twice weekly, with water added as needed.

Embryos were divided into two oxic treatment groups, one hypoxic-incubation treatment - $10 \% \mathrm{O}_{2}$ ('H10') and one normoxic-incubation treatment - 21\% $\mathrm{O}_{2}$ ('N21') acting as control. Hypoxic or normoxic incubation began at $\sim 20 \%$ of development. $10 \% \mathrm{O}_{2}$ is the low value used in previous studies and slightly exceeds the $\sim 85$ torr measured in American crocodile nests (Crocodylus acutus; Lutz and Dunbar-Cooper, 1984). Containers were sealed inside large Ziplock ${ }^{\circledR}$ bags and two holes in the bags allowed parallel inflow and outflow of gas. Oxygen mixtures were set using rotameters or a Sechrist Air-Oxygen mixer (Model 3500HL; Sechrist Industries, Inc. Anaheim, CA, USA), downstream of compressed $\mathrm{N}_{2}$ and air or air alone, with mixtures passing through a $\mathrm{H}_{2} \mathrm{O}$-bubbler to ensure adequate water saturation of $\geq 80-95 \%$ relative humidity. Gas composition was monitored continuously with an oxygen analyzer (S-3AI, Ametek Applied Electrochemistry, IL, USA).

Relative developmental ages used in experiments below (i.e., 70 and 90\%) reflect in ovo developmental stages 24/25 and 27/28, respectively, based on Ferguson (1985; also see Crossley et al. 2003c). Therefore, we studied our two oxic incubation groups at the same developmental stages.

\subsection{Chronic hypoxia with cholinergic and adrenergic blockade, and acute hypoxia with cholinergic or ganglionic blockade}

In a first experiment, at 70\% $(n=5, H 10 ; n=6, N 21), 80 \%(n=7, H 10 ; n=7, N 21)$, and 90\% of development $(n=6, H 10 ; n=6, N 21)$, eggs were removed from their incubators and candled to determine orientation of the embryo and location of an accessible tertiary CAM artery. All eggs were placed in a temperature-controlled surgical chamber $\left(30^{\circ} \mathrm{C}\right)$ under normoxic conditions, and a portion of the eggshell removed under a dissection microscope (Leica MZ6 or MZ3; Leica Microsystems, Waukegan, IL, USA). A tertiary CAM artery was isolated for arterial pressure monitoring and drug injection. An occlusive catheter was inserted into a tertiary CAM artery under a dissection microscope using heat-pulled, heparinized and saline-filled PE 50 tubing, as previously described (Crossley and Altimiras, 2005). The surgical preparations were minimally invasive and durable, lasting for up to 6 hours, and preparations that did not make it through the experiment were discarded from analyses.

Following catheterization, all embryos were transferred to a water-jacketed multi-chamber experimental apparatus ( $\sim 700 \mathrm{~cm}^{3}$ per chamber, one embryo per chamber placed on cotton) and allowed to recover until cardiovascular parameters stabilized, at least for $20 \mathrm{~min}$. Temperature in the chambers was maintained at $30{ }^{\circ} \mathrm{C}$ by recirculating water from a constant temperature circulator (VWR 
International, LLC, West Chester, PA, USA). Each chamber consisted of a container fitted with a lid with three ports that allowed the catheter and airlines to enter the chamber. To prevent changes in chamber temperature due to air inflow, all incoming air traversed a 1-m copper tube submerged within the constant temperature circulator's water bath. This warmed room air was pushed into each chamber from an


$\mathrm{cm}$ above the egg via saline-filled PE 50 tubing, connected to an amplifier, and the pressure signal acquired at $40 \mathrm{~Hz}$ using a Labview ${ }^{\circledR}$ data recording system and software (National Instruments, Austin, TX, USA) or a PowerLab ${ }^{\circledR}$ data recording system (ADInstruments, CO, USA) connected to a computer running Chart ${ }^{\circledR}$ software (v 5.4.2 ADInstruments). Pressure transducers were calibrated prior to each measurement period with a vertical column of saline, and heart rate was determined with a software tachograph based on the arterial pressure trace. Throughout the study, total injection volumes were normalized for each embryonic age to $5 \%$ of total blood volume, $1 / 3$ volume being the drug administered through a $\mathrm{T}$ connector in the arterial catheter line and $2 / 3$ volume being a saline flush to insure the drug had entered the CAM artery (Crossley and Altimiras, 2005). To account for potential effects of the injection volume, all embryos received a control injection of heparinized saline with the volume identical to each injection volume. Subsequent to the control saline injection, in the first experiment cholinergic and adrenergic antagonists were injected in the following sequence: atropine ( $\left.1 \mathrm{mg} \mathrm{kg}^{-1}, \mathrm{SIGMA}\right) \mathrm{a}$ cholinergic antagonist, followed by propranolol ( $3 \mathrm{mg} \mathrm{kg}^{-1}$, SIGMA) a non-specific $\beta$-adrenoreceptor antagonist, and finally phentolamine ( $\left.1 \mathrm{mg} \mathrm{kg}^{-1}, \mathrm{SIGMA}\right)$ a nonspecific $\alpha$-adrenoreceptor antagonist.

In a second experiment, at $70 \%(\mathrm{n}=10, \mathrm{H} 10 ; \mathrm{n}=10, \mathrm{~N} 21)$ and $90 \%$ of development $(\mathrm{n}=12, \mathrm{H} 10$; $\mathrm{n}=8, \mathrm{~N} 21$ ), subsequent to the control saline injection, each embryo was subjected to an acute 5 min hypoxic event before and after ganglionic blockade with hexamethonium. Hypoxic exposure was induced by providing a $10 \% \mathrm{O}_{2}$ mixture to an individual chamber at $9.0 \mathrm{~L} \mathrm{~min}^{-1}$ via a Sechrist mixer. Holes in the experimental chambers were covered during hypoxic exposure, and gas composition in the chamber dropped rapidly to $10 \% \mathrm{O}_{2}$ within 5-10 s. After $5 \mathrm{~min}$, the chamber was flushed with air $\left(200 \mathrm{ml} \mathrm{min}^{-1}\right)$. Embryos were allowed to recover from hypoxic exposure for at least $30 \mathrm{~min}$. Second, embryos were injected with hexamethonium $\left(25 \mathrm{mg} \mathrm{kg}^{-1}\right)$. After a $30 \mathrm{~min}$ period to allow the drug to react, the embryos were exposed to $10 \% \mathrm{O}_{2}$ a second time for $5 \mathrm{~min}$.

In a third experiment, at 70\% $(n=5, H 10 ; n=9, N 21)$ and $90 \%$ of development $(n=6, H 10 ; n=$ $7, \mathrm{~N} 21$ ), subsequent to the control saline injection, each embryo was subjected to an acute 5 min hypoxic event before and after cholinergic blockade with atropine. First, embryos were exposed to hypoxic gas for $5 \min \left(10 \% \mathrm{O}_{2} ; 9.0 \mathrm{~L} \mathrm{~min}^{-1}\right)$ and allowed to recover for at least $30 \mathrm{~min}$, secondly embryos were injected 
with atropine $\left(1 \mathrm{mg} \mathrm{kg}^{-1}\right)$ and allowed to recover for at least $30 \mathrm{~min}$, and finally embryos were exposed to $10 \% \mathrm{O}_{2}$ a second time for $5 \mathrm{~min}$.

\subsection{Chronic hypoxia and circulating catecholamines}

In $70 \%(n=5, H 10 ; n=9, N 21), 80 \%(n=5, H 10 ; n=13, N 21)$, and $90 \%$ of development embryos $(\mathrm{n}=2, \mathrm{H} 10 ; \mathrm{n}=7, \mathrm{~N} 21)$, a $1 \mathrm{ml} \mathrm{CAM}$ arterial blood sample was obtained through a tertiary CAM artery, as previously described (Crossley and Altimiras 2005). Blood samples were mixed with 5 $\mu \mathrm{L}$ of an EGTA/glutathione solution $(0.2 \mathrm{M} / 0.2 \mathrm{M})$ to prevent catecholamine oxidation and immediately spun down to separate the plasma. Samples were maintained at $-70{ }^{\circ} \mathrm{C}$ until analysis was carried out (within one month). HPLC analysis of plasma catecholamines was carried out as previously described (Fritsche and Nilsson, 1990).

\subsection{Data analysis and presentation}

Absolute or transformed mean CAM arterial pressure (MAP; $\mathrm{kPa})$ and mean heart rate $\left(\mathrm{f}_{\mathrm{H}}\right.$; beats

$\min ^{-1}$ ) represent the grand mean of mean values from individual embryos. Control MAP and $\mathrm{f}_{\mathrm{H}}$, prior to any acute hypoxic exposure or drug injection, were taken from concurrent 5-10 min periods of stable pressure and heart rate. Acute hypoxia $\left(10 \% \mathrm{O}_{2}\right)$ causes an almost immediate bradycardia in alligator embryos (see Eme et. al., 2011b), and reponses to all acute hypoxic challenges were taken from the last minute of the 5-minute exposure to $10 \% \mathrm{O}_{2}$. Following completion of each trial, embryos were euthanized with an overdose of isoflurane (Isoflo; Abbott laboratories, North Chicago, IL, USA), and embryonic wet mass (excluding embryonic membranes) determined to the nearest $\mathrm{mg}$ using an analytical balance (Mettler Toledo XS204). Absolute blood pressure was corrected for the pressure transducer's distance above the egg by adding the pressure corresponding to a water column of the distance from the transducer to the catheter to the recorded pressure $(\mathrm{kPa})$. For comparisons of the magnitude of a change in heart rate or blood pressure between oxic incubation conditions and developmental ages, arcsine square root transformed fractions were used. The magnitude of the effect of hypoxia was expressed in relative terms as the absolute change divided by the absolute control level. Because these values are expressed as percentages, data were transformed (arcsine square root) before statistical analysis. Within each developmental age, a $t$ test was used to compare body mass between oxic incubation treatments. Throughout the text, means are given \pm standard error of the mean (SEM). All statistical significance was determined based on $\alpha=0.05$ (Statistica v9.0; StatSoft, Tulsa, OK, USA). 
2.4.1 Chronic hypoxia with cholinergic and adrenergic blockade, and acute hypoxia with cholinergic or ganglionic blockade

MAP and $\mathrm{f}_{\mathrm{H}}$ responses to atropine, propranolol, phentolamine or hexamethonium were taken for 15-20 min stable periods at least 15-20 min following injection, an acceptable time period for responses to the drugs above (see Crossley and Altimiras, 2000). Within each developmental age and incubation condition, paired $t$ tests were used to compare control MAP and $\mathrm{f}_{\mathrm{H}}$ levels to the responses to drug injection (atropine, propranolol, phentolamine, hexamethonium) in order to determine if each individual

drug had an effect on pressure or heart rate; all embryos within each protocol were treated identically (i.e., received the identical sequence of drugs and/or hypoxia), and, therefore, the paired $t$ test is appropriate (Eme et al., 2011 b). Intrinsic $f_{H}$, after injection with atropine, propranolol and phentolamine, were compared between and within developmental age and oxic incubation condition with a 2-way ANOVA, and a post-hoc SNK separated values into distinct subsets.

\subsubsection{Chronic hypoxia and circulating catecholamines}

Within each developmental age, Mann-Whitney $U$ tests were used to analyze differences in the levels of catecholamines (noradrenaline and adrenaline) between each oxic incubation group. Between each successive developmental age, a Mann-Whitney $U$ test was used to analyze differences in the levels of catecholamines (noradrenaline and adrenaline) within each oxic incubation group.

\section{Results}

As previously shown in alligator embryos, control injections of saline did not alter CAM arterial pressure or heart rate (paired $t$ tests $\mathrm{P}>0.1$; Crossley et al., 2003c; Eme et al., 2011b). For baseline values prior to any manipulation, compared to N21 (normoxic-incubated) embryos, H10 (hypoxic-incubated) embryos were hypotensive at $90 \%$ of development only and bradycardic at 70, 80, and 90\% of development (e.g., Table 1; separate 2-way ANOVAs for pressure or heart rate; $\mathrm{SNK} \alpha=0.05, \mathrm{P}<0.0001)$. In addition, H10 embryos' body mass was significantly less than N21 embryo body mass within 70\% (11.2 \pm 0.7 g vs. 15.1 $\pm 1.2 \mathrm{~g}), 80 \%(17.5 \pm 1.0 \mathrm{~g}$ vs. $27.0 \pm 2.4 \mathrm{~g})$ and $90 \%$ of development $(21.6 \pm 1.2 \mathrm{~g}$ vs. $34.3 \pm 2.0 \mathrm{~g}$; separate $t$ tests, $\mathrm{P}<0.01)$. 


\section{Table 1}

For normoxic $\left(21 \% \mathrm{O}_{2} ; \mathrm{N} 21\right)$ and hypoxic-incubated $\left(10 \% \mathrm{O}_{2} ; \mathrm{H} 10\right)$ alligator embryos at 70 and $90 \%$ of development, CAM arterial pressure (MAP; $\mathrm{kPa})$ and heart rate $\left(\mathrm{f}_{\mathrm{H}}\right.$; beats $\mathrm{min}^{-1}$ ) before (Pre Hex) and after hexamethonium injection (Post Hex). Data are presented as mean $\pm \mathrm{SEM}$.

\begin{tabular}{cccccc}
\hline $\begin{array}{c}\text { Developmental } \\
\text { Age (\%) }\end{array}$ & Variable & $\begin{array}{c}\text { N21 Pre Hex } \\
(\mathrm{n})\end{array}$ & $\begin{array}{c}\text { N21 Post Hex } \\
(\mathrm{n})\end{array}$ & $\begin{array}{c}\text { H10 Pre Hex } \\
(\mathrm{n})\end{array}$ & $\begin{array}{c}\text { H10 Post Hex } \\
(\mathrm{n})\end{array}$ \\
\hline 70 & $\mathrm{MAP}$ & $0.70 \pm 0.05$ & $0.65 \pm 0.05 *$ & $0.70 \pm 0.04$ & $0.69 \pm 0.03$ \\
& & $(10)$ & $(10)$ & $(10)$ & $(10)$ \\
90 & $\mathrm{MAP}$ & $1.47 \pm 0.11$ & $1.37 \pm 0.09$ & $1.04 \pm 0.07^{+}$ & $0.96 \pm 0.07 *$ \\
& & $(7)$ & $(7)$ & $(12)$ & $(12)$ \\
70 & $\mathrm{f}_{\mathrm{H}}$ & $85 \pm 3$ & $86 \pm 3$ & $78 \pm 2+$ & $79 \pm 2$ \\
& & $(10)$ & $(10)$ & $(10)$ & $(10)$ \\
90 & $\mathrm{f}_{\mathrm{H}}$ & $82 \pm 1$ & $83 \pm 1$ & $70 \pm 2+$ & $73 \pm 4$ \\
& & $(7)$ & $(7)$ & $(12)$ & $(12)$ \\
\hline
\end{tabular}

*Hexamethonium injection moderately reduced MAP at 70\% of development in normoxic-incubated embryos and at $90 \%$ of development in hypoxic-incubated embryos (Pre Hex vs. Post Hex; paired $t$ test; $\mathrm{P}<0.05$ ), but did not significantly alter any other responses.

For baseline values prior to any manipulation, compared to N21 embryos, H10 embryos were hypotensive at $90 \%$ of development and bradycardic at 70 and $90 \%$ of development.

\subsection{Chronic hypoxia with cholinergic and adrenergic blockade, and acute hypoxia with cholinergic or ganglionic blockade}

For N21 embryos, injection of atropine had no significant effect on CAM MAP or $\mathrm{f}_{\mathrm{H}}$ at any point during development (Fig. 1; paired $t$ tests $\mathrm{P}>0.20$ ). Following atropine, propranolol injection caused significant hypertensive bradycardia at 70, 80 and 90\% of development in N21 embryos (Fig. 2; paired $t$ tests $\mathrm{P}<0.01$ ). At $90 \%$ of development, propranolol caused a maximum $22 \%$ increase in MAP (Fig. 2a), and a maximum $37.5 \%$ decrease in $f_{H}$ (Fig. 2b). The subsequent injection of phentolamine, after atropine and propranolol, caused a significant hypotension at 70, 80 and 90\% of development (Fig. 3a; maximum $42 \%$ at $90 \%$ of development; paired $t$ tests $\mathrm{P}<0.05$ ), but only caused a significant bradycardia at $70 \%$ of development (Fig. 3b; paired $t$ tests $\mathrm{P}<0.05$ ).

The magnitude of changes in CAM MAP and $\mathrm{f}_{\mathrm{H}}$ in response to atropine (Fig. 4), propranolol (Fig. 5) and phentolamine (Fig. 6) for H10 embryos was overall similar to the changes observed for N21 embryos. There was one notable exception; at $80 \%$ of development, $\mathrm{H} 10$ embryos showed a significantly greater bradycardia in response to propranolol injection (following atropine injection) compared to N21 embryos (Fig. 5b; 2-way ANOVA for heart rate on arc sine square root transformed fractional responses; SNK $\alpha=0.05, \mathrm{P}<0.001)$.

Upon completion of the injections of atropine, propranolol and phentolamine combined, intrinsic $\mathrm{f}_{\mathrm{H}}$ was significantly lower than the pre-injection control values in both the H10 and N21 groups at all 


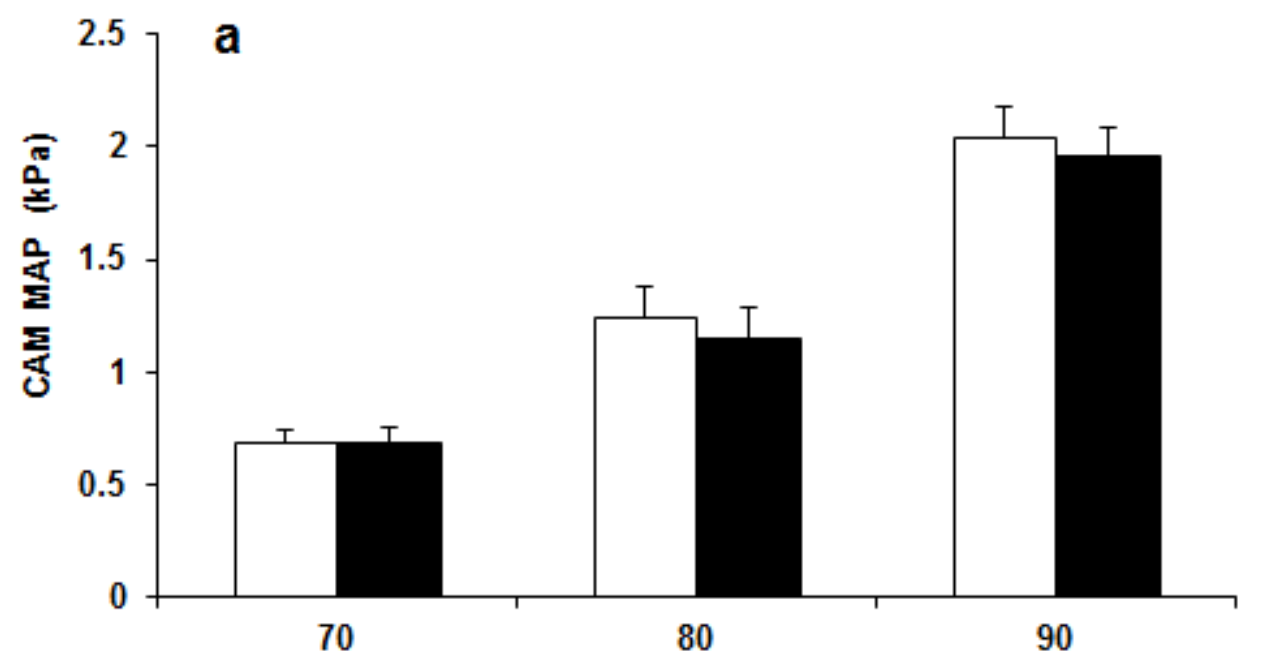

Developmental Age (\% development)

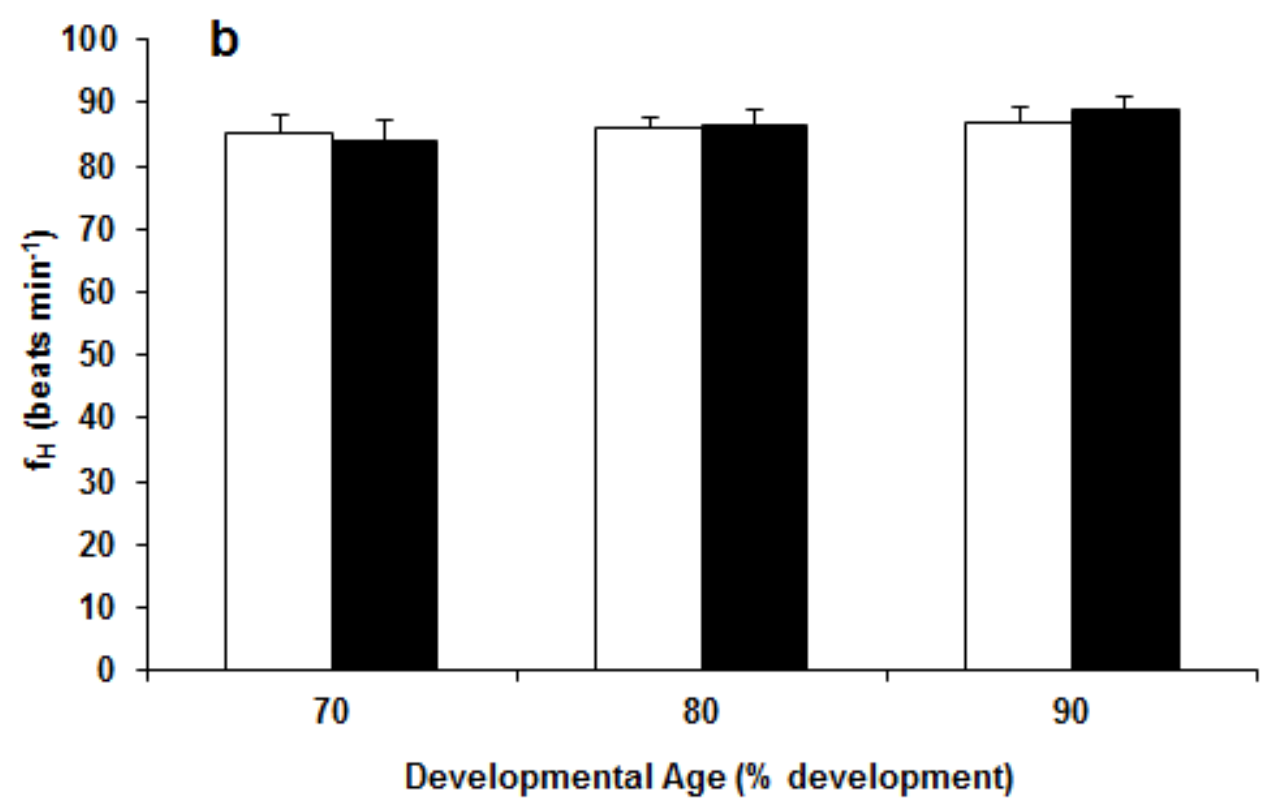

Fig. 1. For normoxic-incubated $\left(21 \% \mathrm{O}_{2} ; \mathrm{N} 21\right)$ alligator embryos at $70 \%(\mathrm{n}=6), 80 \%(\mathrm{n}=7)$, and $90 \%$ of development $(n=6)$, mean CAM arterial pressure (a) and heart rate (b) responses to atropine injection alone. White bars indicate pressure or heart rate response prior to atropine injection, and black bars indicate the responses to atropine injection. Atropine injection did not cause a significant change in pressure or heart rate within each developmental age (paired $t$ tests, $\mathrm{P}>0.20$ ), and atropine injection did not cause a significant relative change in pressure or heart rate across development (separate 2-way ANOVAs for pressure or heart rate on arc sine square root transformed fractional responses, $\mathrm{P}>0.10$ ). Error bars are SEM. 


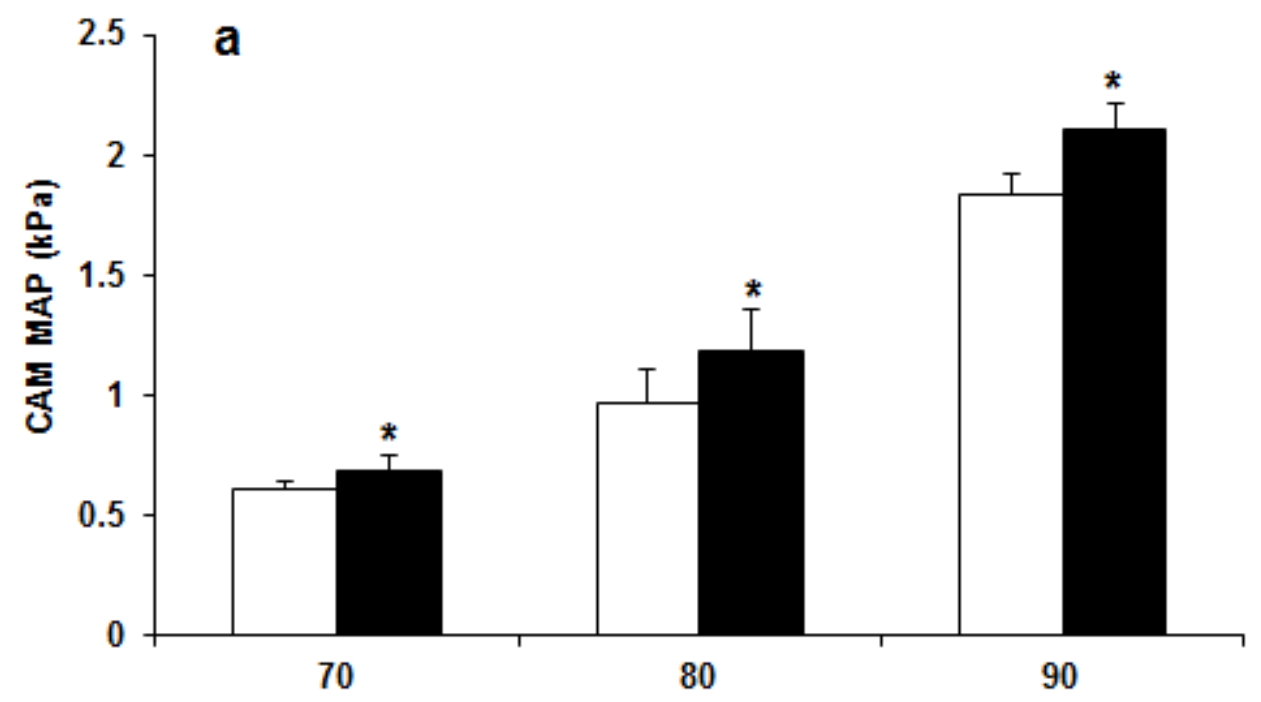

Developmental Age (\% development)

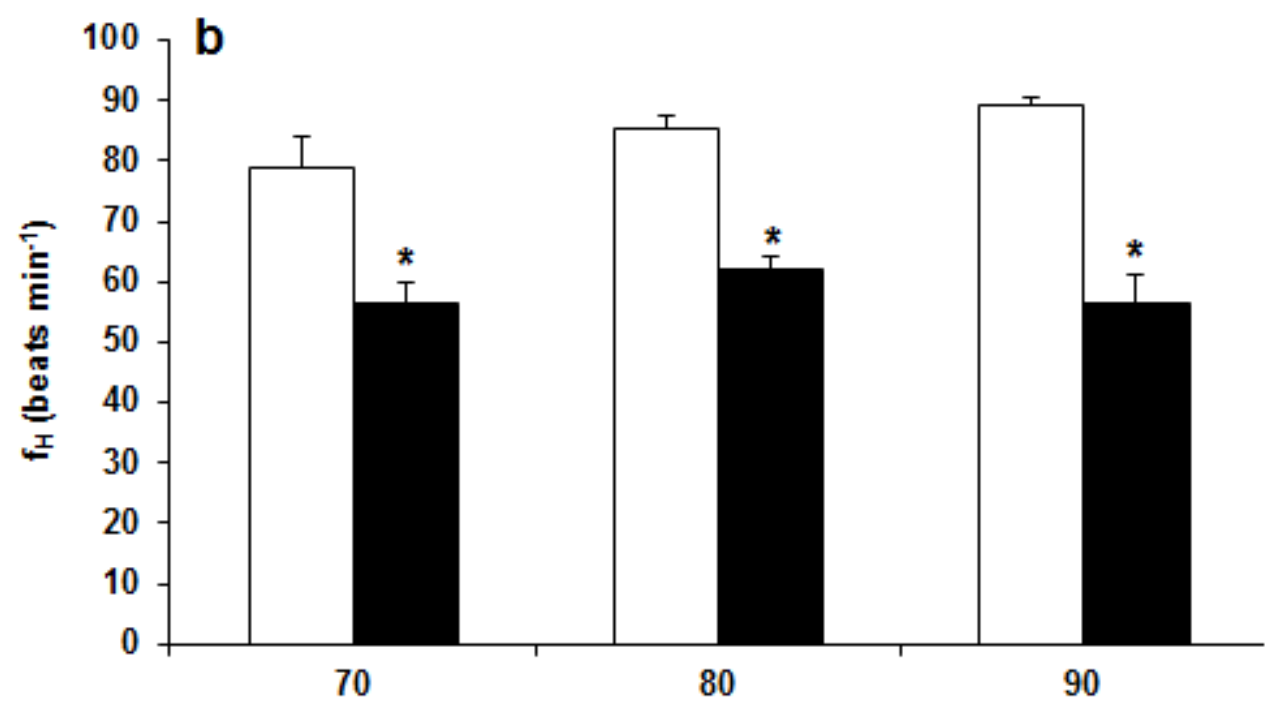

Developmental Age (\% development)

Fig. 2. For normoxic-incubated $\left(21 \% \mathrm{O}_{2} ; \mathrm{N} 21\right)$ alligator embryos at $70 \%(\mathrm{n}=6), 80 \%(\mathrm{n}=7)$, and $90 \%$ of development $(n=6)$, mean CAM arterial pressure $(\mathbf{a})$ and heart rate $(\mathbf{b})$ responses to propranolol injection (after atropine injection). White bars indicate pressure or heart rate response prior to propranolol injection, and black bars indicate the responses to propranolol injection. Propranolol caused a significant hypertension (paired $t$ test, $\mathrm{P}<0.05$ ) and bradycardia (paired $t$ test, $\mathrm{P}<0.01$ ) at all developmental ages. However, propranolol injection did not cause a significant relative change in pressure or heart rate across development (separate 2-way ANOVAs for pressure or heart rate on arc sine square root transformed fractional responses, $\mathrm{P}>0.05)$. Error bars are SEM. 


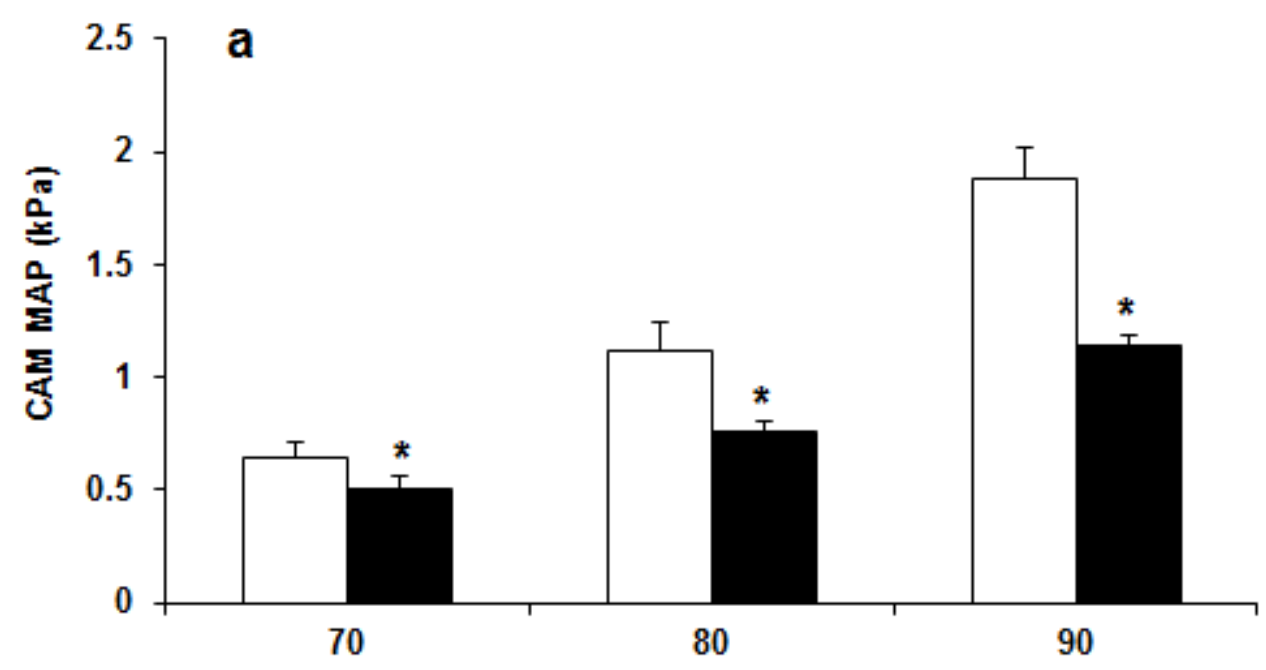

Developmental Age (\% development)



Fig. 3. For normoxic-incubated $\left(21 \% \mathrm{O}_{2} ; \mathrm{N} 21\right)$ alligator embryos at $70 \%(\mathrm{n}=6), 80 \%(\mathrm{n}=7)$, and $90 \%$ of development $(\mathrm{n}=6)$, mean CAM arterial pressure (a) and heart rate $(\mathbf{b})$ responses to phentolamine injection (after sequential atropine and propranolol injection). White bars indicate pressure or heart rate response prior to phentolamine injection, and black bars indicate the responses to phentolamine injection. Phentolamine caused a significant hypotension at all developmental ages (paired $t$ test, $\mathrm{P}<0.01$ ), and phentolamine caused a significant bradycardia at $70 \%$ of development only (paired $t$ test, $\mathrm{P}<0.01$ ). However, phentolamine injection did not cause a significant relative change in pressure or heart rate across development (separate 2-way ANOVAs for pressure or heart rate on arc sine square root transformed fractional responses, $\mathrm{P}>0.05$ ). Error bars are SEM. 

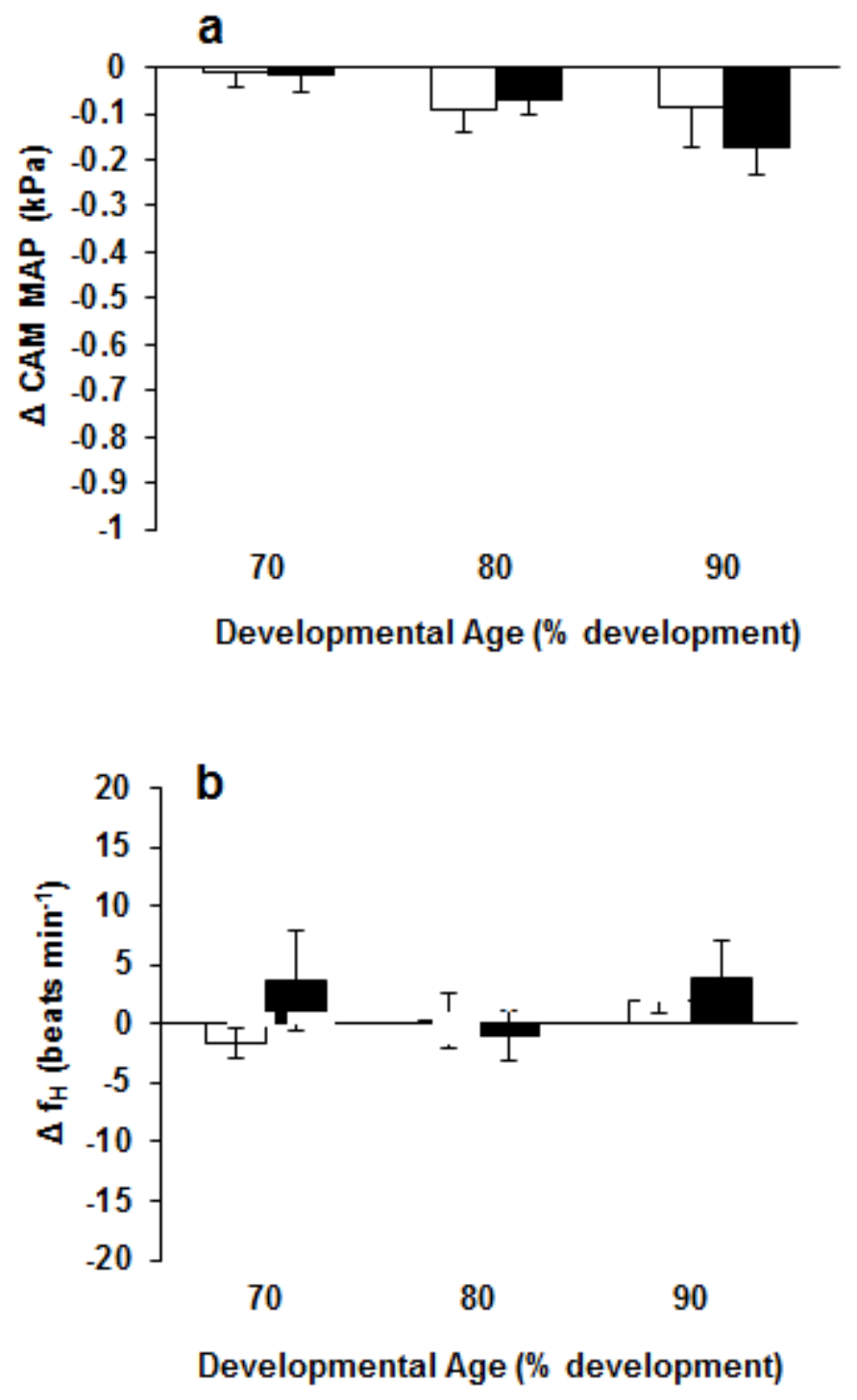

Fig. 4. For normoxic $\left(21 \% \mathrm{O}_{2} ; \mathrm{N} 21\right)$ and hypoxic-incubated $\left(10 \% \mathrm{O}_{2} ; \mathrm{H} 10\right)$ alligator embryos at $70 \%(\mathrm{n}=$ $5, \mathrm{H} 10 ; n=6, \mathrm{~N} 21), 80 \%(\mathrm{n}=7, \mathrm{H} 10 ; \mathrm{n}=7, \mathrm{~N} 21)$, and $90 \%$ of development $(\mathrm{n}=6, \mathrm{H} 10 ; \mathrm{n}=6, \mathrm{~N} 21)$, mean change in CAM arterial pressure (a) and heart rate (b) responses to atropine injection. White bars indicate N21 embryos' change in pressure or heart rate in response to atropine injection, and black bars indicate H10 embryos' change in pressure or heart rate in response to atropine injection. Atropine injection did not cause a significant relative change in pressure or heart rate across development, between N21 and H10 embryos (separate 2-way ANOVAs for pressure or heart rate on arc sine square root transformed fractional responses, $\mathrm{P}>0.10$ ). Error bars are $\mathrm{SEM}$. 

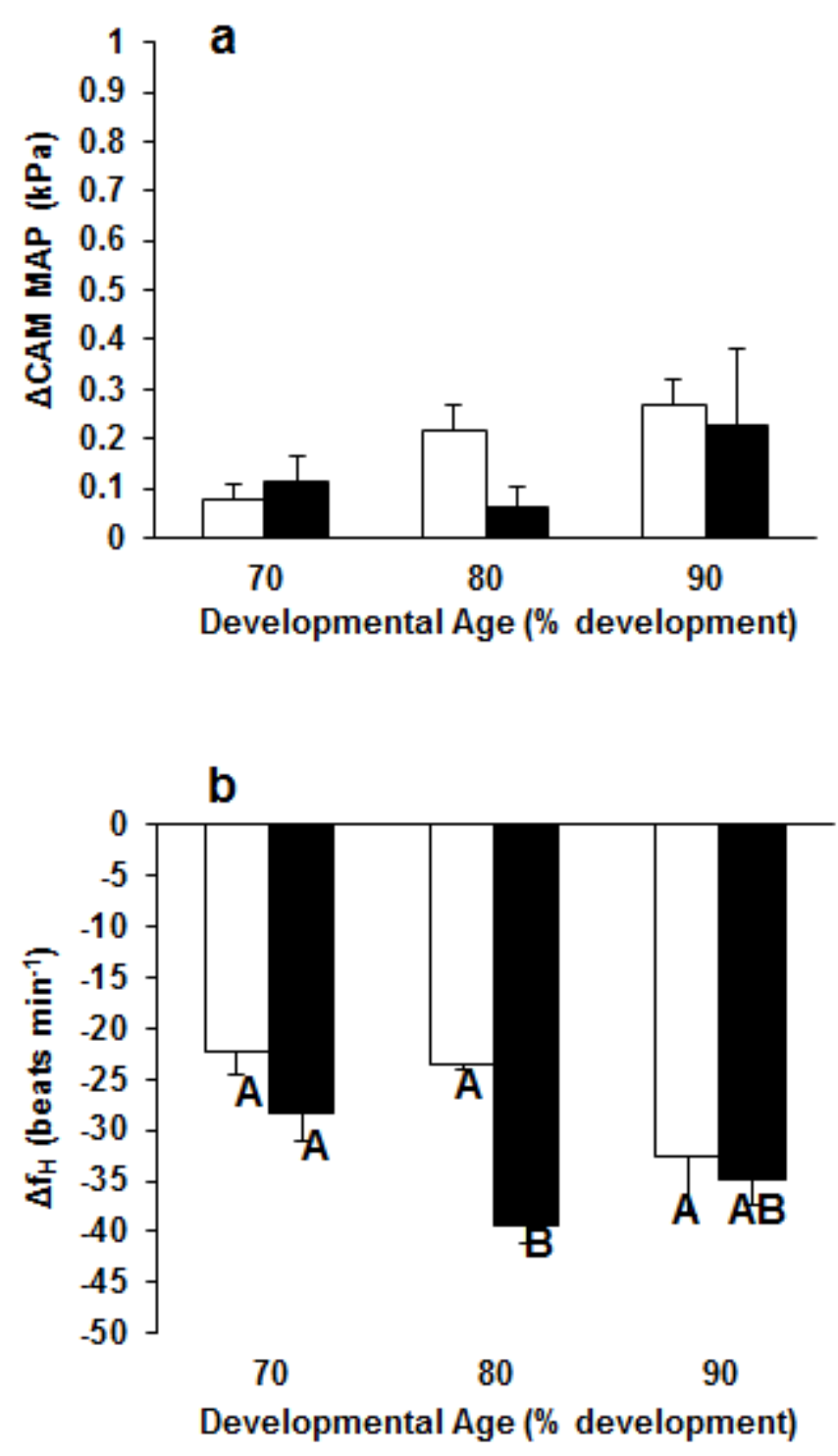

Fig. 5. For normoxic $\left(21 \% \mathrm{O}_{2} ; \mathrm{N} 21\right)$ and hypoxic-incubated $\left(10 \% \mathrm{O}_{2} ; \mathrm{H} 10\right)$ alligator embryos at $70 \%(\mathrm{n}=$ $5, \mathrm{H} 10 ; \mathrm{n}=6, \mathrm{~N} 21), 80 \%(\mathrm{n}=7, \mathrm{H} 10 ; \mathrm{n}=7, \mathrm{~N} 21)$, and $90 \%$ of development $(\mathrm{n}=6, \mathrm{H} 10 ; \mathrm{n}=6, \mathrm{~N} 21)$, mean change in CAM arterial pressure (a) and heart rate (b) responses to propranolol injection (after atropine injection). White bars indicate N21 embryos' change in pressure or heart rate in response to propranolol injection, and black bars indicate $\mathrm{H} 10$ embryos' change in pressure or heart rate in response to propranolol injection. Propranolol injection did not cause a significant relative change in pressure across development, between N21 and H10 embryos (2-way ANOVA for pressure on arc sine square root transformed fractional responses, $\mathrm{P}>0.05$ ). However, propranolol injection did cause a significantly larger bradycardic response for $\mathrm{H} 10$ embryos at $80 \%$ development (2-way ANOVA for heart rate on arc sine square root transformed fractional responses; $\mathrm{SNK} \alpha=0.05, \mathrm{P}<0.001_{\mathrm{A}, \mathrm{AB}, \mathrm{B}}$. Uppercase, bold letters denote SNK post-hoc comparisons of fractional responses of heart rate to propranolol injection between N21 and H10 embryos and between developmental ages. Similar letters indicate fractional responses are not significantly different, and dissimilar letters indicate fractional responses are significantly different. Error bars are SEM. 



Fig. 6. For normoxic $\left(21 \% \mathrm{O}_{2} ; \mathrm{N} 21\right)$ and hypoxic-incubated $\left(10 \% \mathrm{O}_{2} ; \mathrm{H} 10\right)$ alligator embryos at $70 \%(\mathrm{n}=$ $5, \mathrm{H} 10 ; \mathrm{n}=6, \mathrm{~N} 21), 80 \%(\mathrm{n}=7, \mathrm{H} 10 ; \mathrm{n}=7, \mathrm{~N} 21)$, and $90 \%$ of development $(\mathrm{n}=6, \mathrm{H} 10 ; \mathrm{n}=6, \mathrm{~N} 21)$, mean change in CAM arterial pressure (a) and heart rate (b) responses to phentolamine injection (after sequential atropine and propranolol injection). White bars indicate N21 embryos' change in pressure or heart rate in response to phentolamine injection, and black bars indicate $\mathrm{H} 10$ embryos' change in pressure or heart rate in response to phentolamine injection. Phentolamine injection did not cause a significant relative change in pressure or heart rate across development, between N21 and H10 embryos (separate 2way ANOVAs for pressure or heart rate on arc sine square root transformed fractional responses, $\mathrm{P}>0.05$ ). Error bars are SEM.

points of development studied (Fig. 7; 2-way ANOVA for heart rate; $\operatorname{SNK} \alpha=0.05, \mathrm{P}<0.01$ ). The post injection, after atropine, propranolol, and phentolamine, values for $\mathrm{f}_{\mathrm{H}}$ differed from the $\mathrm{N} 21$ group at $80 \%$ and $90 \%$ of development with a $43 \%$ and 59\% lower value, respectively (Fig. 7; 2-way ANOVA for heart rate; $\mathrm{SNK} \alpha=0.05, \mathrm{P}<0.01)$.

Hexamethonium injection only slightly reduced MAP at $70 \%$ of development in N21 embryos (decrease 7\%) and in 90\% of development $\mathrm{H} 10$ embryos (decrease 8\%; Table 1). Hexamethonium 
injection did not have a major impact on the response to acute hypoxic exposure (Table 2). Atropine injection did not significantly alter the response to acute hypoxic exposure (Table 3; separate 2-way ANOVAs for heart rate or blood pressure on arc sine square root transformed fractional responses, $\mathrm{P}>0.1$.).

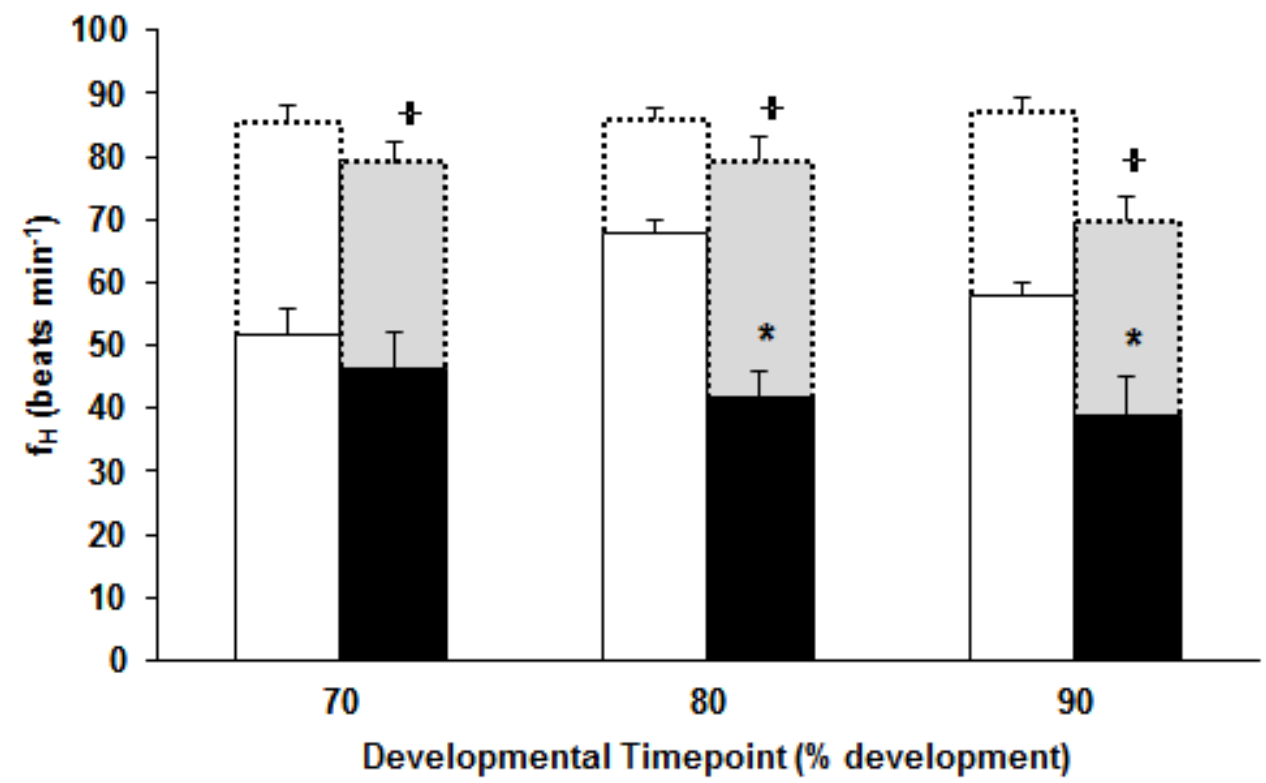

Fig. 7. For normoxic $\left(21 \% \mathrm{O}_{2} ; \mathrm{N} 21\right)$ and hypoxic-incubated $\left(10 \% \mathrm{O}_{2} ; \mathrm{H} 10\right)$ alligator embryos at $70 \%(\mathrm{n}=$ $5, \mathrm{H} 10 ; \mathrm{n}=6, \mathrm{~N} 21), 80 \%(\mathrm{n}=7, \mathrm{H} 10 ; \mathrm{n}=7, \mathrm{~N} 21)$, and $90 \%$ of development $(\mathrm{n}=6, \mathrm{H} 10 ; \mathrm{n}=6, \mathrm{~N} 21)$, baseline (dotted bars) and intrinsic (solid bars) heart rate. Dotted white bars indicate N21 embryos' baseline heart rate prior to any drug injection, and dotted grey bars indicate H10 embryos' baseline heart rate. For baseline values, compared to N21 embryos, H10 embryos were bradycardic at 70 and $90 \%$ of development ( +2 -way ANOVA for heart rate; SNK $\alpha=0.05, \mathrm{P}<0.0001$ ). Solid white bars indicate N21 embryos' intrinsic heart rate following sequential injection of atropine, propranolol and phentolamine, and solid black bars indicate $\mathrm{H} 10$ embryos' intrinsic heart rate. Intrinsic $\mathrm{f}_{\mathrm{H}}$ was significantly lower than the pre-injection control values in both $\mathrm{N} 21$ and $\mathrm{H} 10$ embryos at each developmental age (2-way ANOVA for heart rate; SNK $\alpha=0.05, \mathrm{P}<0.01$ ). Intrinsic $\mathrm{f}_{\mathrm{H}}$ in $\mathrm{H} 10$ embryos was significantly lower than N21 embryos' at $80 \%$ and $90 \%$ of development (Fig. 7; *2-way ANOVA for heart rate; SNK $\alpha=0.05$, $\mathrm{P}<0.01)$. Error bars are SEM. 


\section{Table 2}

For normoxic $\left(21 \% \mathrm{O}_{2} ; \mathrm{N} 21\right)$ and hypoxic-incubated $\left(10 \% \mathrm{O}_{2} ; \mathrm{H} 10\right)$ alligator embryos at 70 and $90 \%$ of development, mean absolute change in CAM arterial pressure $(\triangle \mathrm{MAP} ; \mathrm{kPa})$ and heart rate $\left(\Delta \mathrm{f}_{\mathrm{H}}\right.$; beats $\left.\mathrm{min}^{-1}\right)$ in response to acute hypoxic exposure $\left(10 \% \mathrm{O}_{2}, 5 \mathrm{~min}\right)$ before (Pre Hex) and after hexamethonium injection (Post Hex). Data are presented as mean \pm SEM.

\begin{tabular}{cccccc}
\hline $\begin{array}{c}\text { Developmental } \\
\text { Age (\%) }\end{array}$ & Variable & $\begin{array}{c}\text { N21 Acute Hypoxia } \\
\text { Pre Hex } \\
(\mathrm{n})\end{array}$ & $\begin{array}{c}\text { N21 Acute Hypoxia } \\
\text { Post Hex } \\
(\mathrm{n})\end{array}$ & $\begin{array}{c}\text { H10 Acute Hypoxia } \\
\text { Pre Hex } \\
(\mathrm{n})\end{array}$ & $\begin{array}{c}\text { H10 Acute Hypoxia } \\
\text { Post Hex } \\
(\mathrm{n})\end{array}$ \\
\hline 70 & $\Delta \mathrm{MAP}$ & $0.05 \pm 0.02$ & $0.06 \pm 0.02 *$ & $0.06 \pm 0.02 *$ & $0.03 \pm 0.02$ \\
& & $(10)$ & $(10)$ & $(10)$ & $(10)$ \\
90 & $\Delta \mathrm{MAP}$ & $0.19 \pm 0.06 *$ & $0.19 \pm 0.05 *$ & $0.12 \pm 0.04 *$ & $0.07 \pm 0.05$ \\
& & $(7)$ & $(7)$ & $(7)$ & $(7)$ \\
70 & $\Delta \mathrm{f}_{\mathrm{H}}$ & $-6 \pm 5$ & $-11 \pm 2 *$ & $-8 \pm 2 *$ & $-9 \pm 5$ \\
& & $(10)$ & $-8 \pm 10$ & $-12)$ & $(12)$ \\
90 & $\Delta \mathrm{f}_{\mathrm{H}}$ & $-12 \pm 2 *$ & $(7)$ & $-6 \pm 3$ & $-7 \pm 3 *$ \\
& & $(7)$ & $(7)$ & $(7)$ \\
\hline
\end{tabular}

*Significant differences from baseline values immediately prior to acute hypoxic exposure (paired $t$ test, $\mathrm{P}<0.05)$.

\section{Table 3}

For normoxic $\left(21 \% \mathrm{O}_{2} ; \mathrm{N} 21\right)$ and hypoxic-incubated $\left(10 \% \mathrm{O}_{2} ; \mathrm{H} 10\right)$ alligator embryos at 70 and $90 \%$ of development, mean absolute change in CAM arterial pressure $(\triangle \mathrm{MAP} ; \mathrm{kPa})$ and heart rate $\left(\Delta \mathrm{f}_{\mathrm{H}}\right.$; beats $\left.\min ^{-1}\right)$ in response to acute hypoxic exposure $\left(10 \% \mathrm{O}_{2}, 5 \mathrm{~min}\right)$ before (Pre Atropine) and after atropine injection (Post Atropine). Data are presented as mean $\pm \mathrm{SEM}$.

\begin{tabular}{cccccc}
\hline $\begin{array}{c}\text { Developmental } \\
\text { Age (\%) }\end{array}$ & $\begin{array}{c}\text { Oxic } \\
\text { Treatment (n) }\end{array}$ & $\begin{array}{c}\Delta \text { PAP } \\
\text { Pre Atropine }\end{array}$ & $\begin{array}{c}\Delta \text { MAP } \\
\text { Post Atropine }\end{array}$ & $\begin{array}{c}\Delta \mathrm{f}_{\mathrm{H}} \\
\text { Pre Atropine }\end{array}$ & $\begin{array}{c}\Delta \mathrm{f}_{\mathrm{H}} \\
\text { Post Atropine }\end{array}$ \\
\hline 70 & $\mathrm{~N} 21(9)$ & $0.09 \pm 0.02$ & $0.05 \pm 0.03$ & $-10 \pm 1$ & $-9 \pm 3$ \\
90 & $\mathrm{~N} 21(7)$ & $0.371 \pm 0.02$ & $0.32 \pm 0.07$ & $-13 \pm 4$ & $-10 \pm 2$ \\
70 & $\mathrm{H} 10(5)$ & $0.10 \pm 0.05$ & $-0.12 \pm 0.06$ & $3 \pm 1$ & $-8 \pm 2$ \\
90 & $\mathrm{H} 10(6)$ & $0.19 \pm 0.07$ & $0.10 \pm 0.07$ & $-5 \pm 4$ & $-5 \pm 1$ \\
\hline
\end{tabular}

Atropine did not alter responses to acute hypoxia, separate 2-way ANOVAs for heart rate or blood pressure on arc sine square root transformed fractional responses, $\mathrm{P}>0.1$. 


\subsection{Chronic hypoxia and circulating catecholamines}

Plasma noradrenaline levels changed maximally by $150 \%$ in the $\mathrm{N} 21$ group over the period of development studied (Table 4). This change was significant ( ${ }^{\top} \mathrm{P}<0.01$ ) at 90\% of development compared to $80 \%$ of development (Table 4), while H10 group was unchanged between 80 and $90 \%$ of development (Table 4). The H10 group had significantly higher levels of plasma noradrenaline (184\% and 100\% higher respectively) at $70 \%$ and $80 \%$ of development compared to the $\mathrm{N} 21$ group $(\mathrm{P}<0.04$; Table 4$)$.

Plasma adrenaline levels increased $380 \%$ during the course of the study in the N21 group with a significant increase at $80 \%$ of development compared to $70 \%$ of development (Table 4 ). The H10 group had significantly higher levels of plasma adrenaline (280\% higher) at $70 \%$ of development compared to the N21 group ( $\mathrm{P}<0.04$; Table 4).

\section{Table 4}

For normoxic $\left(21 \% \mathrm{O}_{2} ; \mathrm{N} 21\right)$ and hypoxic-incubated $\left(10 \% \mathrm{O}_{2} ; \mathrm{H} 10\right)$ alligator embryos at 70,80 and $90 \%$ of development, mean noradrenaline (NA) and adrenaline (A) concentrations. Data are presented as mean \pm SEM.

\begin{tabular}{cccc}
\hline $\begin{array}{c}\text { Developmental } \\
\text { Age (\%) }\end{array}$ & $\begin{array}{c}\text { Oxic } \\
\text { Treatment (n) }\end{array}$ & NA (nM) & A (nM) \\
\hline 70 & N21 (9) & $50.9 \pm 12.2$ & $7.2 \pm 2.4$ \\
70 & H10 (5) & $145.1 \pm 32.3^{*}$ & $24.7 \pm 11.1^{*}$ \\
80 & N21 (13) & $75.7 \pm 14.3$ & $19.7 \pm 5.5^{+}$ \\
80 & H10 (5) & $151.4 \pm 20.8^{*}$ & $35.0 \pm 15.7^{+}$ \\
90 & N21 (7) & $236.3 \pm 46.8^{*}$ & $29.2 \pm 11.0$ \\
90 & H10(2) & $191.3 \pm 98.0$ & $45.8 \pm 32.4$
\end{tabular}

*Within a given developmental time point, between oxic incubation treatments, H10 embryos showed significantly higher levels of NA and A at $70 \%$ of development, as well as NA at $80 \%$ of development only (Mann-Whitney $U$ test; $\mathrm{P}<0.04$ ).

${ }^{+}$Across development, within oxic incubation treatments, mean levels of NA increased significantly between 80 and $90 \%$ of development in the N21 group (Mann-Whitney $U$ test; $\mathrm{P}<0.01$ ).

${ }^{+}$Across development, within oxic incubation treatments, mean levels of A increased significantly between 70 and $80 \%$ of development in the H10 and N21 groups (Mann-Whitney $U$ test; $\mathrm{P}<0.01$ ).

\section{Discussion}

The responses shown in this study to cholinergic, ganglionic and adrenergic blockade in alligator embryos were very similar to previously reported responses of domestic chicken embryos (Gallus gallus 
and domesticus; e.g., Crossley and Altimiras, 2000; Tazawa et al., 1992) and demonstrated an asynchrony between the development of cholinergic and adrenergic tone. Cholinergic blockade with atropine injection did not cause a significant change in heart rate or arterial pressure or alter the generally depressive effects of acute hypoxia in embryonic alligator. Ganglionic blockade with hexamethonium also did not have a major impact on baseline cardiovascular variables in alligator embryos in the present study, similar to chicken embryos (Crossley and Altimiras, 2000). However, the $\beta$-adrenergic antagonist propranolol induced a bradycardia of similar magnitude at 70,80 and 90\% of alligator development, as well as a hypertension of similar magnitude at all developmental ages studied, indicating that like embryonic chicken, embryonic alligators display an important cardiac and vascular $\beta$-adrenergic tone (Crossley and Altimiras, 2000). Finally, $\alpha$-adrenergic blockade with phentolamine caused a hypotension during the final $\sim 30 \%$ of incubation in embryonic alligator, very similar to embryonic chicken (Crossley and Altimiras, 2000). Therefore, embryonic alligator and domestic chicken both rely on adrenergic control of resting cardiovascular function, however chicken embryos, and not alligator embryos, have in some cases displayed cholinergic tone from the autonomic nervous system late in development (see Andrewartha et al., 2011 for review). In alligator embryos, the adrenergic tone originates entirely from circulating catecholamines, as ganglionic blockade with hexamethonium did not have a major impact on baseline heart rate, similar to chicken embryos (Tazawa et al., 1992; Crossley and Altimiras, 2000). In addition, embryonic emu (Dromiceius novaehollandiae) has a cholinergic tone beginning at $\sim 70 \%$ of development. Like chicken and alligator, however, embryonic emu display similar, constant adrenergic blockade responses through the last third of incubation (Crossley et al., 2003a). The differences observed clearly demonstrate that the limited number of species studied restricts our capacity to identify common features of cardiovascular regulatory development in archosaurs. However, data to date suggest that basal archosaurs (crocodilians) do not have functional autonomic tone in ovo, whereas avian archosaurs have some components of cholinergic tone late in development.

Autonomic tone did not contribute to maintenance of $\mathrm{f}_{\mathrm{H}}$ and MAP in either N21 or H10 embryonic alligators over the final 30\% of incubation, refuting our hypothesis that chronic hypoxic incubation (H10 group) would accelerate development of resting cholinergic or adrenergic tone in embryonic alligators. This is in contrast to the relatively early onset of autonomic nervous system (ANS) control in fetal mammals, as well as the central role of the ANS in response to hypoxia during mammalian development (e.g. Giussani et al., 1993; Giussani et al., 1994). As shown previously when measured in normoxia (21\% $\mathrm{O}_{2}$ ), $\mathrm{H} 10$ embryos showed depressed resting $\mathrm{f}_{\mathrm{H}}$ and MAP when compared to N21 embryos, and acute hypoxia $\left(10 \% \mathrm{O}_{2}, 5 \mathrm{~min}\right)$ caused a transient bradycardia in both oxic groups (Crossley and Altimiras, 2005; Eme et al., 2011b). While adrenergic and cholinergic tones in embryonic alligators were unaffected 
by chronic hypoxia, it remained a possibility that $\alpha$-tone, $\beta$-tone or cholinergic tone was transiently stimulated and differed when embryos were acutely challenged with hypoxia $\left(10 \% \mathrm{O}_{2}, 5 \mathrm{~min}\right)$. To investigate these scenarios, we exposed embryos to acute hypoxia before and after ganglionic blockade with hexamethonium, as well as before and after cholinergic blockade with atropine. Ganglionic and cholinergic blockade did not alter resting cardiovascular levels and had no effect on the bradycardic response to acute hypoxia in either N21 or H10 embryos. In day 21 embryonic chicken, hypoxic-induced bradycardia is stimulated by acetylcholine release from vagal nerve terminals (Crossley et al., 2003b); however, we show that this is not the case for embryonic alligator at 70-90\% of development. Similar acute responses to hypoxic exposure, following ganglionic and cholinergic blockade, clearly suggest that the depressive cardiovascular effects of hypoxia on embryonic alligator are independent of the autonomic nervous system and its receptors.

The mechanisms accounting for the chronic bradycardia and hypotension in H10 alligator embryos measured in normoxia were not absolutely identified in this study; however, it is possible that increased circulating catecholamines account for some of the effects. Circulating levels of noradrenaline increased from 70 to $90 \%$ of development in both $\mathrm{N} 21$ and H10 embryos, which likely accounts for the increase in $\beta$-adrenoreceptor tone on $\mathrm{f}_{\mathrm{H}}$ during this period (Fig. 5), and chronic hypoxic incubation altered plasma catecholamine levels of H10 compared to N21 embryos. Importantly, H10 embryos showed significantly higher levels of noradrenaline and adrenaline at 70\%, as well as higher noradrenaline at $80 \%$ of development compared to N21 embryos, but levels were equal at $90 \%$. While our ability to emphasize the significance of the trend for increased catecholamines in $\mathrm{H} 10$ embryos is limited by the small sample size at $90 \%$ of incubation, data suggest hypoxic incubation shifts the timing of maximal plasma catecholamine levels to an earlier point in incubation. In addition, propranolol injection caused a significantly larger bradycardic response for $\mathrm{H} 10$ embryos at 80\% development in the present study (Fig 5 b). Chronically elevated levels of catecholamines may alter the normal balance between $\alpha$ and $\beta$ adrenoreceptors' stimulation in H10 alligator embryos, a speculation based on data from several previous studies. Regional hypoxia increases vascularization of the CAM in alligators (Corona and Warburton, 2000), and this increase in the amount of parallel vascular beds could cause decreased chorioallantoic resistance and pressure (Crossley and Altimiras, 2005). Given this physical alteration, chronic overstimulation of $\beta$-adrenoreceptors may be necessary to maintain adequate cardiac output and perfusion, while balancing the need to maintain adequate peripheral resistance. Stimulation of $\beta$ adrenoreceptors on the heart is responsible for maintaining embryonic cardiac output, and H10 embryos have previously shown increased blood flow to the CAM (Eme et al., 2011a), suggesting that overstimulation of $\beta$-adrenoreceptors may be partially responsible for increased CAM blood flow. In 
addition, chronic hypoxic incubation of chickens results in increased cardiac and vascular sensitivity to $\beta$ stimulation (Lindgren and Altimiras, 2009; Lindgren et al., 2011), as well as increased plasma noradrenaline (Lindgren et al., 2011). Altered adrenoreceptor stimulation balance may also require differential density of $\alpha$-adrenoreceptors in H10 embryos, as previously discussed in regards to adrenergic maturation of 'N21' alligators (Crossley et al., 2003c), or the lack of these (functional) receptors in the CAM. Chicken embryo CAM arteries are insensitive to $\alpha$-adrenergic stimulation, suggesting that $\alpha$ adrenoreceptors are absent from, or not active in, this large vascular bed in chicken embryos (Lindgren et al., 2010) and possibly alligator embryos. If overall CAM resistance is lowered in hypoxia via increased $\beta$-adrenoreceptor stimulation, not offset by $\alpha$-adrenoreceptor stimulation, this could lead to relative blood pooling the CAM and a reduction in venous return, which could account for the chronic bradycardia observed in H10 embryos during measurement in normoxia.

However, reduced arterial oxygenation has been shown to have direct action(s) on the heart, and this could account for the observed bradycardia in H10 embryos measured during normoxia, as well as the bradycardia during acute hypoxia. Acute hypoxia significantly reduces sinoatrial pacemaker rates in neonatal guinea pigs (as much as adult rates), and the neonatal pacemaker responds with a blunted rate increase following adrenaline injection compared to the adult pacemaker (Stowe et al., 1985). Similarly, isolated rabbit pacemaker cells from the sinoatrial node showed decreased pacemaker rates when exposed to ischemia-like conditions (Du and Nathan, 2007). In H10 alligator embryos, complete cholinergic and adrenergic blockade resulted in lowered intrinsic heart rates at 80 and $90 \%$ of development, relative to N21 embryos (Fig 7). This suggests that chronic hypoxia may have changed the density of channels for conducting the sinoatrial pacemaker's action potentials. In addition, altered sinoatrial conductivity could cause the acute bradycardic response to hypoxia, as well as the chronic bradycardia of H10 embryos measured in normoxia, possibly through hyperpolarization of pacemaker cells caused by the opening of ATP-sensitive $\mathrm{K}^{+}$channels (Han et al., 1996). It is possible that the hearts of H10 embryos had lowered sinoatrial membrane permeability, and future studies should examine molecular and histological changes in the hearts of hypoxic-incubated alligator embryos, and include in situ studies of sinoatrial pacemaker activities in response to acute and chronic hypoxia.

This study demonstrates that while adrenergic receptor tone on the cardiovascular system is present in embryonic alligators, it is not originating from the sympathetic nervous system. Further, this tonic stimulation is unaffected by chronic, marked developmental hypoxia $\left(10 \% \mathrm{O}_{2}\right)$, suggesting that adrenergic receptor tone is a relatively non-plastic feature of crocodilian development. Recently, we assessed components involved in hypoxic-incubated embryonic alligators' response to phenylbiguanide, a $5-\mathrm{HT}_{3}$ (serotonin) receptor agonist that can stimulate vagal pulmonary $\mathrm{C}$-fiber afferents and a 
'chemoreflex' (Eme et al., 2011b). In that study, we demonstrated that a reflex loop consisting of a ligand binding to $5-\mathrm{HT}_{3}$ receptors, which then transduces an afferent vagal signal to the central nervous system, was blunted in $\mathrm{H} 10$ alligator embryos (Eme et al., 2011b). Chronic hypoxic incubation could have decreased $5-\mathrm{HT}_{3}$ receptor densities, delayed their development, or altered their sensitivity. It is plausible that in response to chronic hypoxia, various crocodilian receptor types respond differently, and that delayed development of function is a possible response to developmental hypoxia (Eme et al., 2011b), but that accelerated development, as originally hypothesized in the present study, is less likely.

Similarly, Crocodylus porosus embryos exposed to hypoxia did not show altered blood-oxygen binding properties (Grigg et al., 1993), suggesting that crocodilian embryos have limited ability to accelerate hematological mechanisms to improve tissue oxygenation during hypoxia. Taken together, the absence of accelerated functional changes in hematological, cholinergic and adrenergic function, as well as the plateau in catecholamine levels approaching hatching, suggest that crocodilian embryos do not alter these pathways to change metabolism or embryo oxygenation. Unlike birds and mammals, the low metabolic rate and lower incubation temperature for crocodilian embryos may preclude any functional need to accelerate development of such variables. To date, only the likely interconnected increased vascularization of the CAM and increased blood flow to the CAM in hypoxic alligator embryos seem likely to aid in increased embryo oxygenation (Corona and Warburton, 2000; Eme et al., 2011a).

\subsection{Summary}

American alligator embryos show asynchrony between the development of cholinergic and adrenergic tone, with adrenergic tone appearing throughout the latter third of development, whereas cholinergic tone is absent up through $90 \%$ of development. Embryonic alligators display significant cardiac and vascular $\beta$-adrenergic tone and rely on circulating catecholamines to control cardiovascular function. Hypoxic incubation beginning at $20 \%$ of development resulted in higher circulating catecholamine levels in alligator embryos at 70 and $80 \%$ of development. However, hypoxia did not accelerate earlier development of autonomic regulation of the cardiovascular system. Given that the autonomic system is present but not tonically active during the last $30 \%$ of alligator development, it is difficult to assess the long-term impact of this hypoxic challenge on cardiovascular regulation in mature animals. However, our data do suggest that chronic hypoxic challenges promote cardiovascular changes (general bradycardia and hypotension), possibly due to increased circulating catecholamine levels. Lastly, we also suggest the direct effects of hypoxia on the cardiovascular system, independent of an autonomic regulatory mechanism, remains a likely mechanism(s) to be investigated. 


\section{Acknowledgements}

The authors sincerely thank the following individuals for help in these studies: Ruth Elsey for access to alligator eggs and her invaluable, continuing support of our research and Kevin Tate for assistance with embryo care and data collection. This work was supported by NSF Career award IBN IOS-0845741 to DAC and NSF award IOB-0445680 to JWH. JE was supported for part of this study by NSF GK-12 grant number DGE-0638751, and travel monies were provided to JE through a JEB Traveling Fellowship (The Company of Biologists, Ltd.).

\section{References}

Ackerman, R.A., 1980. Physiological and ecological aspects of gas exchange by Sea Turtle eggs. Amer. Zool. 20, 575-583.

Andrewartha, S.J., Tazawa, H., Burggren, W.W., 2011. Embryonic control of heart rate: Examining developmental patterns and temperature and oxygenation influences using embryonic avian models. Resp. Physiol. Neurobiol. DOI:10.1016.j.resp.2011.04.014.

Booth, D.T., 1998. Nest temperature and respiratory gases during natural incubation in the broad-shelled river turtle, Chelodina expansa (Testudinata : Chelidae). Aus. J. Zool. 46, 183-191.

Booth, D.T., 2000. The effect of hypoxia on oxygen consumption of embryonic estuarine crocodiles (Crocodylus porosus). J. Herp. 34, 478-481.

Corona, T.B., Warburton, S.J., 2000. Regional hypoxia elicits regional changes in chorioallantoic membrane vascular density in alligator but not chicken embryos. Comp. Biochem. Physiol. A. $125,57-61$.

Crossley II, D.A., Altimiras, J., 2000. Ontogeny of autonomic control of cardiovascular function in the domestic chicken Gallus gallus. Am. J. Physiol. Regul. Integr. Comp. Physiol. 279, R1091R1098.

Crossley II, D.A., Altimiras, J., 2005. Cardiovascular development in embryos of the American Alligator Alligator mississippiensis: effects of chronic and acute hypoxia. J. Exp. Biol. 208, 31-39.

Crossley II, D.A., Baggatto, B.P., Dzialowski, E.M., Burggren, W.W., 2003a. Maturation of cardiovascular control mechanisms in the embryonic emu (Dromiceius novaehollandiae). J. Exp. Biol. 206, 2703-2710.

Crossley II, D.A., Burggren, W.W., Altimiras, J., 2003b. Cardiovascular regulation during hypoxia in embryos of the domestic chicken Gallus gallus. Am. J. Physiol. Regul. Integ. Comp. Physiol. 284, 
219-226.

Crossley II, D.A., Hicks, J.W., Altimiras, J., 2003c. Ontogeny of baroreflex control in the American Alligator Alligator mississippiensis. J. Exp. Biol. 206, 2895-2902.

Du, Y.M., Nathan, R.D., 2007. Ionic basis of ischemia-induced bradycardia in the rabbit sinoatrial node. J. Mol. Cell. Cardio. 42, 315-325.

Eme, J., Crossley II, D.A., Hicks, J.W., 2011a. Role of the left aortic arch and blood flows in embryonic American alligator (Alligator mississippiensis). J. Comp. Physiol. B. 181, 391-401.

Eme, J., Hicks, J.W., Crossley II, D.A., 2011b. Chronic hypoxic incubation blunts a cardiovascular reflex loop in embryonic American alligator (Alligator mississippiensis). J. Comp. Physiol. B.

DOI:10.1007/s00360-011-0569-z

Ferguson, M.W.J., 1985. Reproductive biology and embryology of the crocodilians. In: Gans, C., Billet, F., Maderson, P. (Eds.), Biology of the reptilia, Vol 14 A. John Wiley and Sons, New York. pp. $329-491$.

Fritsche, R., Nilsson, S., 1990. Autonomic nervous control of blood pressure and heart rate during hypoxia in the cod Gadus morhua. J. Comp. Physiol. B. 160, 287-292.

Giussani, D.A., Spencer, J.A.D., Hanson, M.A., 1994. Fetal cardiovascular reflex responses to hypoxaemia. Fetal. Mat. Med. Rev. 6, 17-37.

Giussani, D.A., Spencer, J.A.D., Moore, P.J, Bennet, L., Hanson, M.A., 1993. Afferent and efferent components of the cardiovascular reflex responses to acute hypoxia in term fetal sheep. J. Physiol. 461, 431-449.

Grigg, G.C., Wells, R.M.G., Beard, L.A., 1993. Allosteric control of oxygen binding by haemoglobin during embryonic development in the crocodile Crocodylus porosus: The role of red cell organic phosphates and carbon dioxide. J. Exp. Biol. 175, 15-32.

Han, X., Light, P.E., Giles, W.R., Franch, R.J., 1996. Identification and properties of an ATP-sensitive K ${ }^{+}$ current in rabbit sino-atrial node pacemaker cells. J. Physiol. Lond. 490, 337-350.

Kam, Y.C., 1993. Physiological effects of hypoxia on metabolism and growth of turtle embryos. Resp. Physiol. 92, 127-138.

Lindgren, I., Altimiras, J., 2009. Chronic prenatal hypoxia sensitizes $\beta$-adrenoreceptors in the embryonic heart but causes postnatal desensitization. Am. J. Physiol. Regul. Integr. Comp. Physiol. 297, R258-R264.

Lindgren, I., Crossley II, D.A., Villamor, E., Altimiras, J., 2011. Hypotension in the chronically hypoxic chicken embryo is related to the $\beta$-adrenergic response of chorioallantoic and femoral arteries and not to bradycardia. Am. J. Physiol. Regul. Integr. Comp. Physiol. in press 
Lindgren, I., Zoer, B., Altimiras, J., Villamor, E., 2010. Reactivity of chicken chorioallantoic arteries, avian homologue of human fetoplacental arteries. J. Physiol. Pharmacol. 61, 619-628.

Lutz, P.L., Dunbar-Cooper, A., 1984. The nest environment of the American Crocodile (Crocodylus acutus). Copeia 1, 153-161.

Miller, N.A., 2008. $\mathrm{PO}_{2}$ in Loggerhead Sea Turtle (Caretta caretta) nests measured using fiber-optic oxygen sensors. Copeia 4, 882-888.

Mulder, A.L.M., Van Golde, J.C., Prinzen, F.W., Blanco C.E., 1998. Cardiac output distribution in response to hypoxia in the chick embryo in the second half of the incubation time J. Physiol. 508, 281-287.

Mulder, A.L.M., Van Goor, C.A, Giussani, D.A., Blanco, C.E., 2001. Adrenergic contribution to the cardiovascular response to acute hypoxemia in the chick embryo. Am. J. Physiol. Regul. Integr. Comp. Physiol. 281, R2004-R2010.

Ruijtenbeek, K., le Noble, F.A.C., Janssen, G.M.J., Kessels, G.C.A., Fazzi, G.E., Blanco, C.E., De Mey, J.G.R., 2009. Chronic hypoxia stimulates periarterial sympathetic nerve development in chicken embryo. Circ.102, 2892-2897.

Seymour, R.S., Vleck, D., Vleck, C.M., 1986. Gas exchange in the incubation mounds of megapode birds. J. Comp. Physiol. B 156, 772-782.

Stowe, D.F., Bosnjak, Z.J., Kampine, J.P., 1985. Effects of hypoxia on adult and neonatal pacemaker rates. Obstet. Gynecol. 66, 649-56.

Tazawa, H., Hashimoto, Y., Doi, K., 1992. Blood pressure and heart rate of chick embryo (Gallus domesticus) within the egg: Responses to autonomic drugs. In: Hill, R.B., Kuwasawa K., McMahon, B.R., Kuramoto, T. (Eds.), Phylogenetic Models in Functional Coupling of the CNS and the Cardiovascular System. Karger, Amsterdam, pp. 86-96. 